\title{
Identificación de actores en un desastre a través de Twitter: caso de estudio Sinabung 2018
}

\author{
Daniel Orlando Martínez Quezada • Robinson Ortiz Sierra ${ }^{b}$ \\ - Juan Guillermo Martínez Canoc - Henry Lamos Díaz
}

\begin{abstract}
Resumen: Twitter se ha convertido en una herramienta importante para conocer en tiempo real lo que sucede en el mundo político, social y económico. Esta plataforma es cada vez más atractiva como medio de comunicación en diferentes tipos de eventos. Puede ser usada en procesos de operaciones logísticas y humanitarias mejorando la comunicación entre los actores involucrados en una situación de desastre natural. En el presente trabajo el enfoque de análisis de redes sociales (ARS) se aplicó a datos generados en Twitter sobre un evento de desastre natural (erupción del volcán Sinabung en 2018), analizando tres actores importantes: usuarios, hashtags y URL. A partir de los análisis se identificaron usuarios, temas y fuentes de información relevantes durante la ocurrencia del desastre. Se ofrece así una vista general de las interacciones y el impacto de los elementos más influyentes durante el evento bajo estudio. Se encontró que tienen una importancia destacada los equipos de noticias, las redes sociales y los centros de investigación. Los hallazgos de este estudio fueron comparados con uno anterior, y se encontraron similitudes significativas. Sin embargo, en nuestro estudio se identificaron nuevos actores del ámbito técnico-académico, que buscaron contribuir y difundir información relevante sobre el evento disruptivo.
\end{abstract}

Palabras clave: Twitter; gestión de desastres; análisis de redes sociales

Recibido: 15 de febrero de 2019 Aceptado: 12 de noviembre de 2019

Disponible en línea: 15 de julio de 2020

Cómo citar: D. O. Martínez Quezada, R. Ortiz Sierra, J. G. Martínez Cano, y H. Lamos Díaz, «Identificación de actores en un desastre a través de Twitter: Caso de estudio SINABUNG 2018», Cien.Ing. Neogranadina, vol. 30, n. 1, pp. 117-132, nov. 2019.

a Universidad Autónoma de Bucaramanga. Correo electrónico: dmartinez582@unab.edu.co ORCID: 0000-0002-9910-1770

b Universidad Industrial de Santander. Correo electrónico: roorsierra_90@live.com ORCID: 0000-0001-8386-8558

c Universidad Industrial de Santander. Correo electrónico: juanguillermo_95@hotmail.com ORCID: 0000-0003-1276-8917

d Universidad Industrial de Santander. Correo electrónico: hlamos@uis.edu.co ORCID: 0000-0003-1778-9768 


\title{
Identifying Actors in a Disaster Using Twitter: Sinabung 2018 Case Study
}

\begin{abstract}
Twitter has become an important tool to learn about political, social, and economic developments in real time. This platform has become increasingly attractive as a means of communication in various situation; for instance, it can be used in logistical and humanitarian operations to improve coordination among actors involved in a natural disaster. In this paper, the social media analytics (SMA) approach is applied to data generated by Twitter about a natural disaster event (eruption of the Sinabung volcano in 2018) using three significant actors: users, hashtags and URLs. As a result, relevant users, topics, and sources of information during the disaster are identified. This provides an overview of the interactions and impact of the most influential elements during the event under study. News agencies, social media, and research centers were found to be of paramount importance. These findings are compared to those of a previous study, revealing substantial similarities. However, this study identifies new actors from the technicalacademic field who sought to contribute to and disseminate relevant information about the disruptive event.
\end{abstract}

Keywords: Twitter; disaster management; social media analytics 


\section{Introducción}

La Agencia Federal de Gestión de Emergencias estadounidense (Fema, por sus siglas en inglés) califica como desastre la ocurrencia de una catástrofe natural, un accidente tecnológico o un evento provocado por el hombre que da lugar a graves daños a propiedades, muertes o heridos múltiples. En este contexto, se entiende la gestión de desastres como el conjunto de procesos diseñados para ser implementados antes, durante y después de los desastres, que permitan prevenir o mitigar sus efectos [1].

La comunicación es un componente central de la gestión de desastres. Dadas las amenazas a la vida humana y la propiedad, las personas necesitan información sobre lo que sucedió y lo que todavía está ocurriendo dentro de un área afectada por un desastre [2]. Debido a que las redes sociales en general, y Twitter en particular, ofrecen una rápida recuperación de información de multitud de fuentes y a su vez son canales de comunicación que permiten llegar a muchas personas [3]-[6], se han desarrollado estudios sobre su uso sistemático como parte de una respuesta a emergencias. El mecanismo de análisis utilizado es la extracción de datos de dichas redes para identificar las necesidades de una comunidad afectada por un desastre [5], [7]. Por otro lado, las redes sociales se han utilizado como medio de comunicación entre organismos de control y usuarios afectados por el desastre con el fin de articular la reacción inmediata ante situaciones de emergencia [8], [9].

Las redes sociales han asumido un rol importante en la comunicación durante eventos catastróficos, por lo cual se han venido revaluado los canales de información (redes sociales vs. medios tradicionales) y fuentes (agencias nacionales vs. locales) en términos de su capacidad para generar resultados públicos deseados (intenciones de buscar y compartir informaciones de emergencia) [10]. Ademas se han analizado las características de las fuentes de información - como individuos (por ejemplo, personalidades, periodistas, autoridades, etc.) y organizaciones (por ejemplo, el gobierno, los medios de comunicación, ONG, etc.) - en el entorno de las redes sociales durante un desastre natural. Para eso se ha examinado un conjunto de factores que describen el comportamiento de dichas fuentes, tales como el tiempo de los trinos, la ubicación y las características del usuario [9].

El análisis de redes sociales (ARS) es una metodología que busca, mediante la aplicación de modelos extraídos de la teoría de grafos, predecir el comportamiento de una red social e identificar las estrategias de los actores que la componen. El ARS surgió por primera vez como un enfoque distintivo del análisis estadístico dentro de la antropología, la sicología social y la sociología. Luego influyó en los desarrollos teóricos y metodológicos en muchas otras áreas de las ciencias sociales. De esta forma, el ARS se vinculó a temas más amplios, lo que promovió la investigación y exploración de las implicaciones de otros modelos matemáticos y estadísticos [11].

Por medio del ARS se describe el comportamiento de una red social a partir de sus aristas y nodos. El análisis de las interacciones en un contexto de gestión de desastres tiene un gran valor para descubrir patrones. Su aplicación a Twitter ha llevado a identificar algunos arquetipos de conversación con sus propias estructuras de comunidad [12]. Además, permite evaluar las oportunidades informativas para individuos o grupos de personas en términos de exposición y control de la información, al tener conocimiento de las rutas existentes de intercambio de esta [13], [14].

La importancia de un sistema de comunicación confiable como parte de una respuesta efectiva ante un desastre natural nos ha llevado a la posibilidad de utilizar la metodología ARS para la identificación de los actores relevantes y fuentes de información a partir de los datos proporcionados por la red social Twitter. Por tanto se establece un caso de estudio (volcán Sinabung 2018), con el fin de implementar dicha metodología para tal identificación. A partir de los resultados obtenidos se identifican los actores (equipos de noticias, centros de investigación, entre otros) que tuvieron mayor impacto en la comunicación en torno al caso a través de Twitter.

La contribución principal de este trabajo se encuentra en el análisis del impacto de usuarios, 
URL y hashtags en los procesos de difusión de información, lo cual no se encuentra en [15]. Los resultados permitieron identificar ciertos comportamientos característicos, como el hecho de que los centros de noticias tienen una influencia importante en el proceso de difusión (retweets) no solo por su participación con contenido propio, sino por la selección y difusión de contenidos de terceras partes. Esto se evidencia en las altas medidas de centralidad de intermediación y valor propio halladas para este tipo de cuentas.

El presente documento se encuentra organizado como sigue: en la sección 1 se describen algunos fundamentos teóricos del ARS, en la sección 2 se detalla la metodología implementada para la realización del estudio, en la sección 3 se hace una descripción del caso y los resultados se discuten en la sección 4. Finalmente, las conclusiones y recomendaciones para trabajos futuros se presentan en la sección 5.

\section{Análisis de redes sociales (ARS)}

El análisis de redes sociales (ARS) es una metodología científica para estudiar, visualizar y cuantificar las relaciones entre los actores de aquellas. La estructura de red es una herramienta útil para representar sistemas de objetos interrelacionados. Una representación matemática adecuada de una red se consigue por medio de un grafo $G$. Un grafo $G$ es una pareja $(V, E)$, con $V$ definido como un conjunto finito no vacío de elementos llamados vértices y $E \subseteq V \times V$ llamados aristas. Un grafo se puede representar por medio de una matriz $A$ con elementos $A_{i j} \in\{0,1\}$, donde la entrada es 1 cuando el vértice $i$ es adyacente a $j$.

En muchas aplicaciones de ARS, el objetivo principal es identificar a los actores más importantes de la red. En tales casos se considera que un nodo de red (un actor) es prominente si está "involucrado" de manera importante con otros nodos que forman la red social. A partir de esta idea se han diseñado diversas medidas de centralidad basadas en parámetros estadísticos para mostrar diferencias en la importancia de los actores [16], [17].
La centralidad de grado total $C_{d}$ es una medida a nivel individual que cuenta el número de vínculos que tiene un nodo. Los individuos con mayor grado de centralidad en la red de colaboración tienen más probabilidades de ser líderes de colaboración, ya que tienen el mayor potencial de ser "conscientes" de los demás. El cálculo de esta métrica se muestra en la ecuación 1.

$C_{\boldsymbol{d}}(i)=d_{\boldsymbol{i}}=\sum_{\boldsymbol{j}} \quad A_{\boldsymbol{i j}} \quad$ (ecuación 1)

La centralidad de cercanía mide la importancia de un vértice por su cercanía a otros vértices. Para esto es necesario el cálculo de la distancia promedio definida como se muestra en la ecuación 2 , donde es el menor número de aristas que hay que recorrer para ir del nodo al nodo. La centralidad de cercanía es el inverso de la distancia y se denota como se muestra en la ecuación 3.

$$
\begin{aligned}
& D_{\text {avg }}(\boldsymbol{i})=\frac{\mathbf{1}}{\boldsymbol{n}-\mathbf{1}} \sum_{\boldsymbol{j} \neq \boldsymbol{i}} \quad g(i, j) \quad \text { (ecuación 2) } \\
& C_{c}=\frac{n-1}{\sum_{j \neq i} g(i, j)} \\
& \text { (ecuación 3) }
\end{aligned}
$$

La centralidad de intermediación o betweenness se usa con el fin de hallar los individuos más importantes para la comunicación y difusión de la información. Se define como el número de rutas más cortas que pasan a través de un vértice, tal como se describe en la ecuación 4, donde es el número de rutas más cortas entre y. Así pues, es el número de rutas más cortas entre y que pasan por .

$C_{B}(i)=\sum_{\boldsymbol{s} \neq \boldsymbol{i} \neq \boldsymbol{t}} \quad G_{\boldsymbol{s t}}(i) \frac{\mathbf{1}}{\boldsymbol{G}_{\boldsymbol{s} \boldsymbol{t}}}, s \quad$ (ecuación 4)

Otra métrica importante para la centralidad es el valor propio, que se determina por la importancia de los "amigos" de este vértice, un indicador que nos permite determinar qué tan importante es el individuo para otros individuos (sus "amigos"). Se define como se muestra en la ecuación 5, que demuestra que la centralidad corresponde al mayor valor propio de la matriz de adyacencia
$C_{E}(i) \sim \sum_{j} \quad A_{i j} C_{E}(i)$
(ecuación 5) 


\section{Metodología}

Para el desarrollo del presente trabajo la metodología constó de tres fases:

- Extracción de datos: los datos utilizados son tomados de la red social Twitter a partir del hashtag \#sinabung, mediante la API de Twitter (https:// developer.twitter.com/).

- Modelación a partir de redes: para el presente trabajo se construyeron tres grafos distintos, uno por cada elemento que se quería analizar en la red -usuarios, URL y hashtags-. El grafo de usuarios está constituido por nodos que representan a los usuarios y aristas que representan la acción de retweet. El modelo para el caso de las URL es un grafo bipartito en el cual los nodos corresponden a las URL y a los usuarios. Cada arista de este grafo es el uso de una URL por parte de un usuario en un trino. En el grafo de hashtags los nodos representan cada hashtag utilizado y las aristas, la aparición simultanea de dos hashtags en una misma publicación.

- Análisis de centralidad: en esta etapa se realiza el cálculo de las medidas de centralidad detalladas en la sección 1 y se analizan los resultados generados identificando los diez nodos más centrales en cada red propuesta.

\section{Caso de estudio}

La erupción del volcán Sinabung se registró en Indonesia el 18 de febrero de 2018 a las 22 horas, con erupciones de ceniza de 5 a 7 kilómetros de altura (figura 1). La erupción alcanzo un área densamente poblada debido a su magnitud (categoría $2^{1}$ ). El análisis del flujo de información generado a través Twitter con ocasión de la emergencia se hizo por medio de la metodología ARS. con el fin de de determinar el comportamiento de los usuarios involucrados en dicho flujo.

1 Correspondiente a erupciones con una altura menor a $10 \mathrm{~km}$.
La muestra de datos de trabajo consta de 42087 trinos publicados del 18 de febrero al 1 de marzo de 2018 (doce días incluyendo el inicio de erupción) generados por 31450 usuarios. Los 42087 trinos están compuestos por 8288 trinos originales y 33799 retweets. En el trabajo se tuvieron en cuenta, como ya se ha dicho, usuarios, hashtags y URL. En los trinos se encontraron más de 939 hashtags distintos y el 85 \% contenía más de 2 hashtags y 102 URL diferentes.

El 18 de febrero se dieron las primeras alertas del volcán. En este día un solo trino se generó poco después de las 22 horas, cuando se empezó a visualizar el fuego eruptivo. Al día siguiente se presenta una mayor cantidad de trinos (770), publicados principalmente por medios de comunicación. El número de publicaciones fue en aumento hasta el 20 de febrero y a partir de allí disminuyó paulatinamente, como se muestra en la figura 2.

\section{Resultados}

El análisis del caso tiene en cuenta tres grafos, uno para los trinos y los otros dos para los hashtags y los URL. En el grafo de la figura 3 se representa la interacción de los usuarios de Twitter durante el evento de estudio a través de los trinos. En este grafo los nodos representan a los 31459 usuarios y los enlaces, la acción de publicar un trino o la de dar retweet. A partir de lo presentado en la figura 3 se puede observar que no todos los nodos se conectan entre sí y que existen grupos muy densos alrededor de algunos de aquellos. A partir de la estructura general de la red se construye el subgrafo presentado en la figura 4, con 264 enlaces y 282 vértices, que considera solo los nodos con centralidad de grado mayor o igual a 3 , es decir, más de tres retweets realizados o recibidos. La elaboración de este subgrafo tiene como propósito facilitar los procesos de cómputo de las medidas de centralidad, lo que permite identificar las interacciones de los usuarios más influyentes en cuanto a la difusión de información durante el periodo del desastre. 

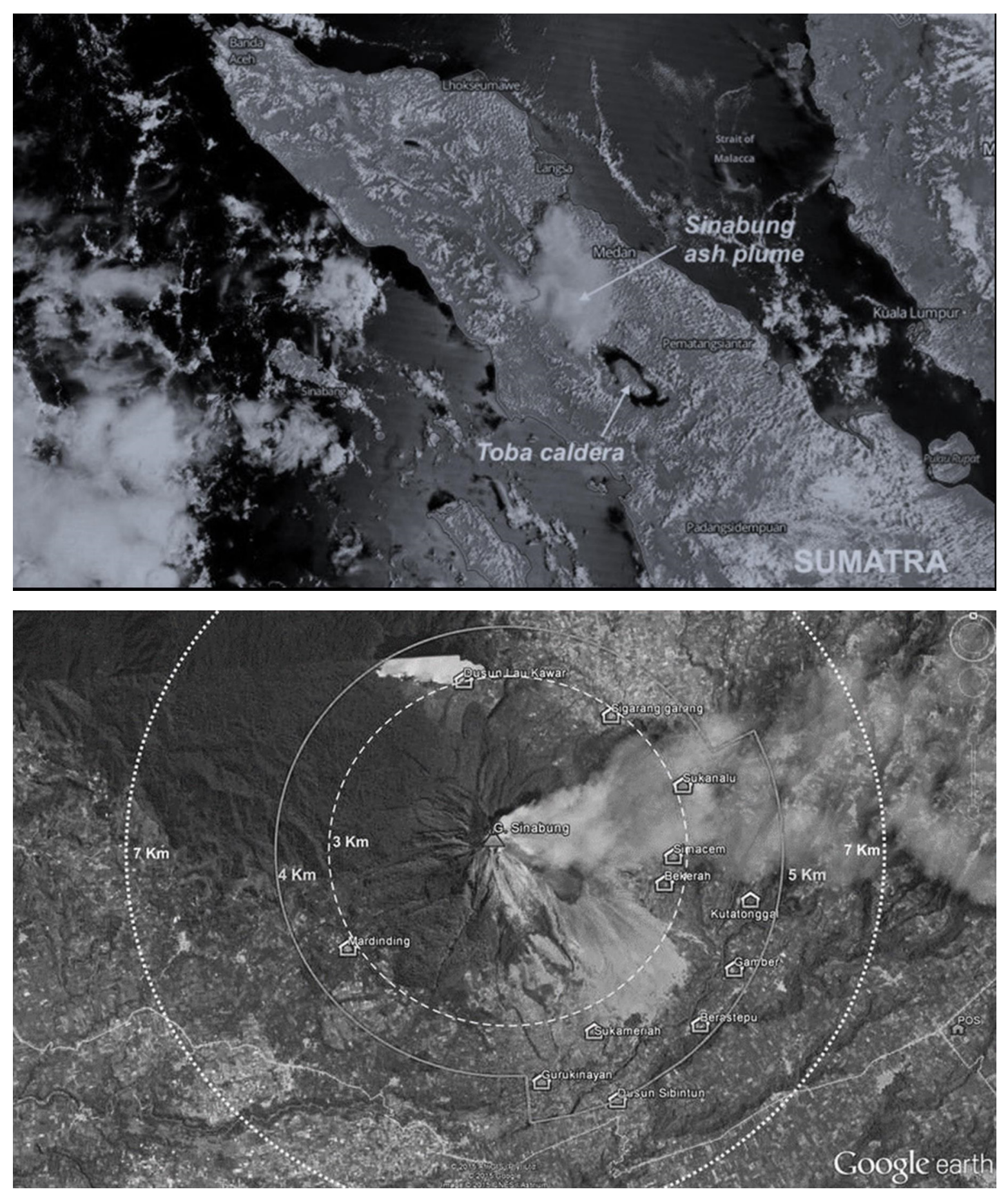

Figura 1. Erupción del volcán Sinabung en la isla de Sumatra

Fuente: [18]. 
Vol. 30(1)

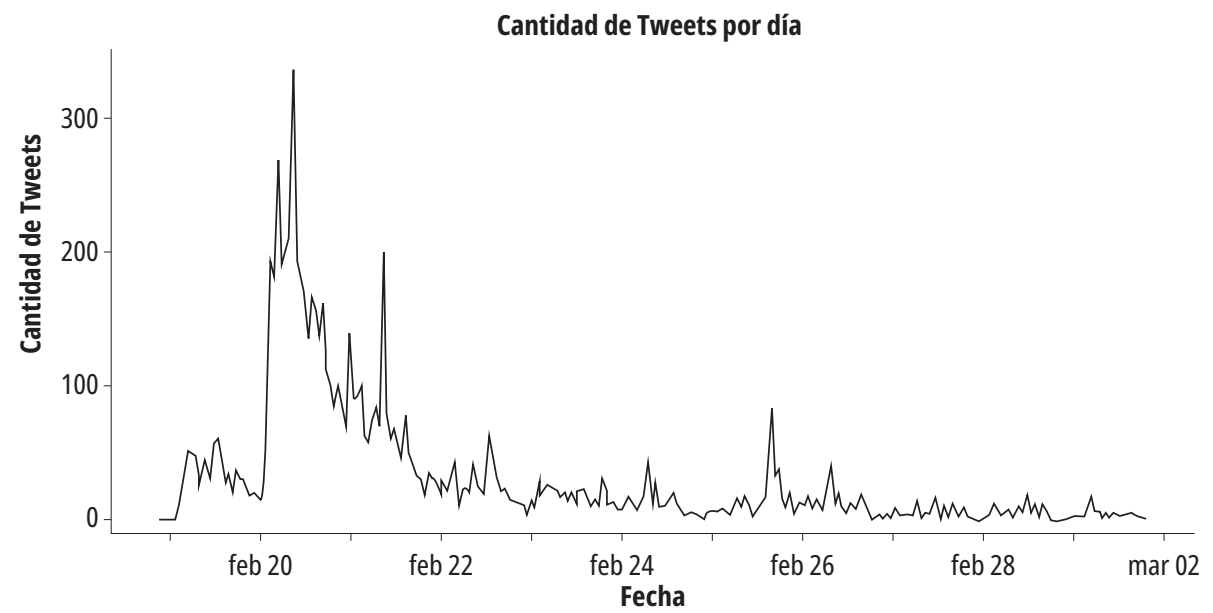

Figura 2. Número de trinos por fecha

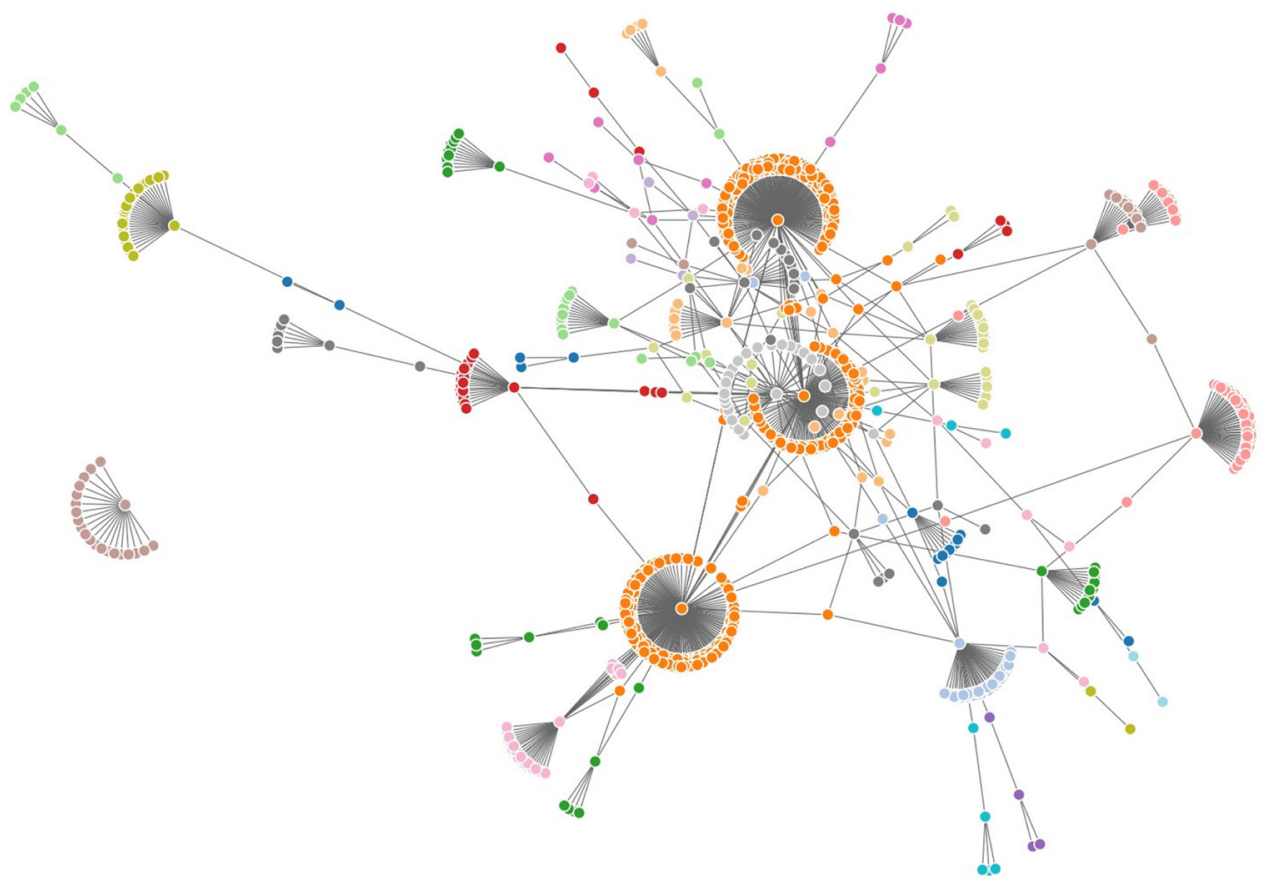

Figura 3. Estructura general de la red de retweets 


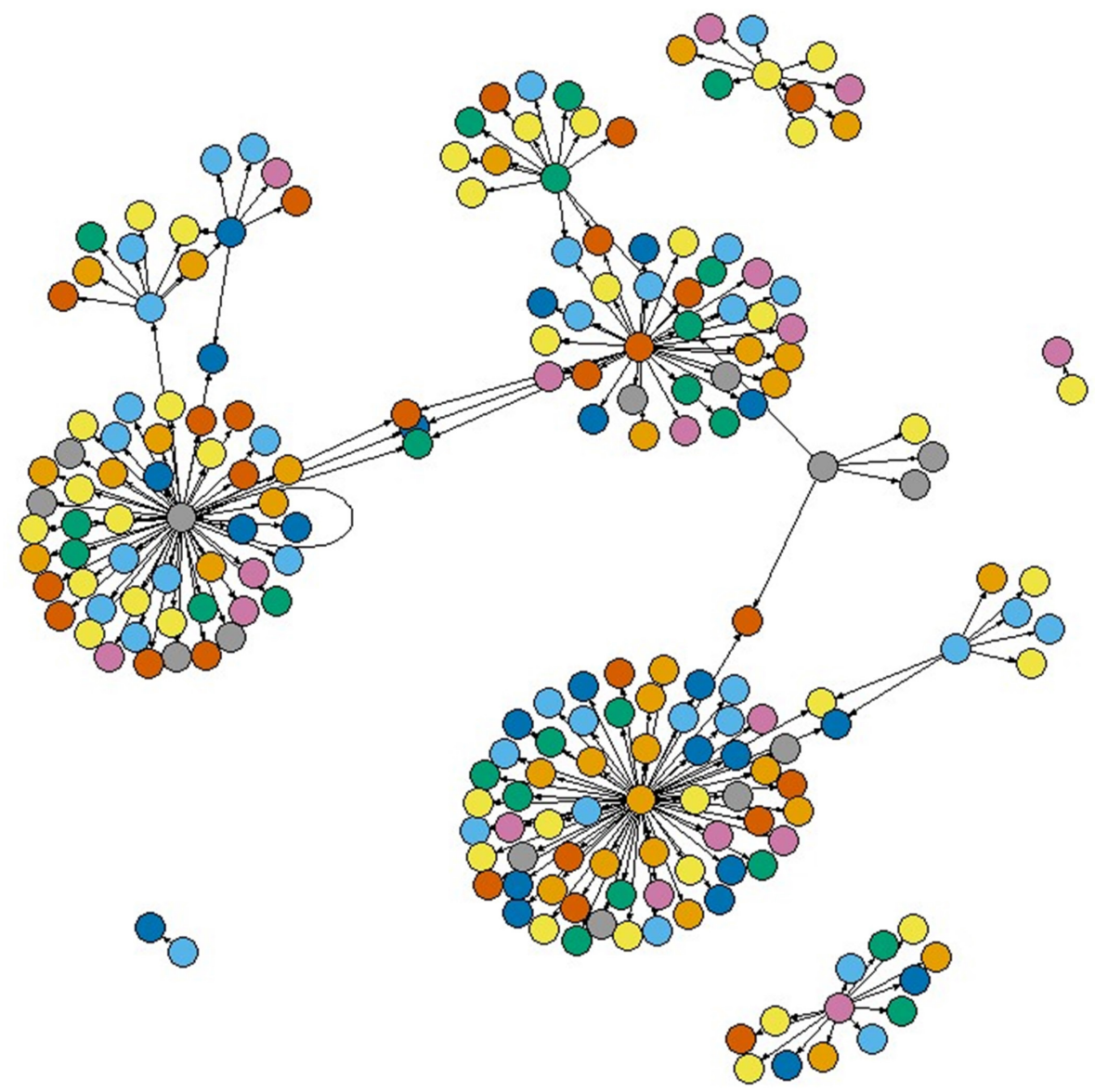

Figura 4. Red de los principales usuarios destacados en los retweets

El cálculo de métricas de centralidad nos permite hallar los actores importantes dentro de la red social. En la tabla 1 se muestran los 10 nodos (usuarios) más importantes de la red analizada, de acuerdo con la centralidad de grado. Dentro de estos se encuentran profesionales técnicos (Chematierra 61, Janinekrippner 34, f_a_h_a_d 9), instituciones gubernamentales para la gestión de desastres (Sutopo_Pn 53, infobencana 7), centros de investigación (severeweatherEU 12, earthquakestime 7, volcanodiscover 6) y portavoces de centros de noticias (Earthuncuttv 13, Karoglobal 9). Los usuarios relacionados con profesionales técnicos enviaron mensajes con el análisis de causas y posibles riesgos involucrados en la situación. Es de resaltar la participación de agencias gubernamentales encargadas de la gestión de desastres. Los mensajes publicados por estos actores se enfocaron en documentar y reportar a la comunidad interesada los sucesos y cambios en el área afectada. Por parte de los centros de investigación y los periodistas se encontraron mensajes que documentaban de manera gráfica (imágenes y videos) el desastre.

La tabla 1 muestra la distribución de grado de los nodos (usuarios), sesgada a la derecha. Solo 5 usuarios, es decir el $1.78 \%$, tienen una centralidad de grado mayor a 10 (solo 5 usuarios hicieron más de 10 retweets), y $96 \%$ muestran una centralidad de grado menor a 5. Esto indica que pocos usuarios se preocuparon por la difusión de los mensajes en la red. 
Tabla 1. Top 10 de centralidad de grado y distribución de grado

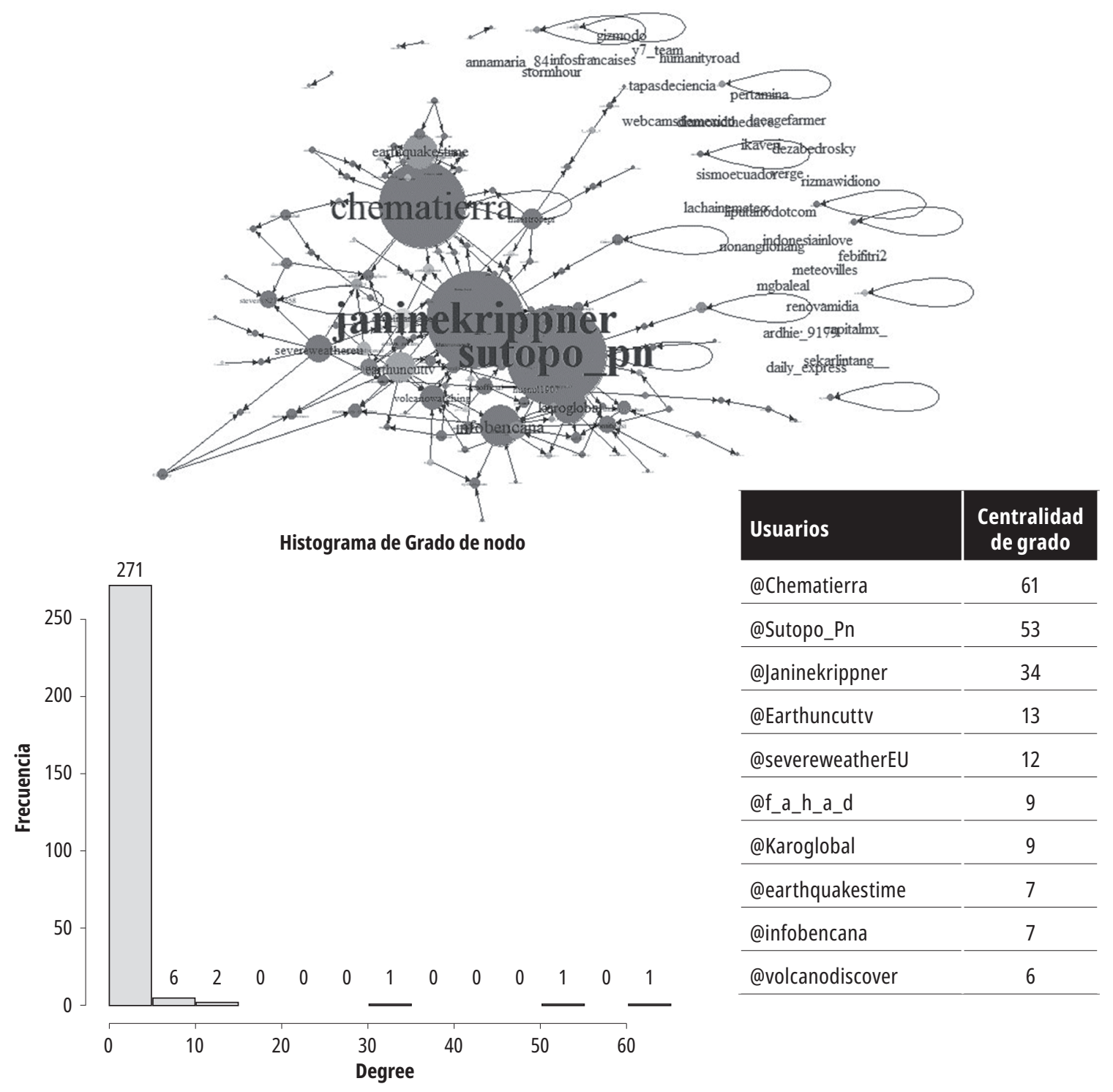

En la tabla 2 se presentan en orden descendente los valores de centralidad de intermediación para 10 usuarios. Los usuarios con mayor centralidad de intermediación se denominan guardianes pues son los que controlan el flujo de información entre las comunidades. Los guardianes @infobencana y @karoglobal corresponden respectivamente a un centro de gestión de desastres y uno de noticias. Sus altos puntajes en la métrica de centralidad de intermediación se deben a que compartieron información de diferentes usuarios. 
Tabla 2. Top 10 centralidad de intermediación
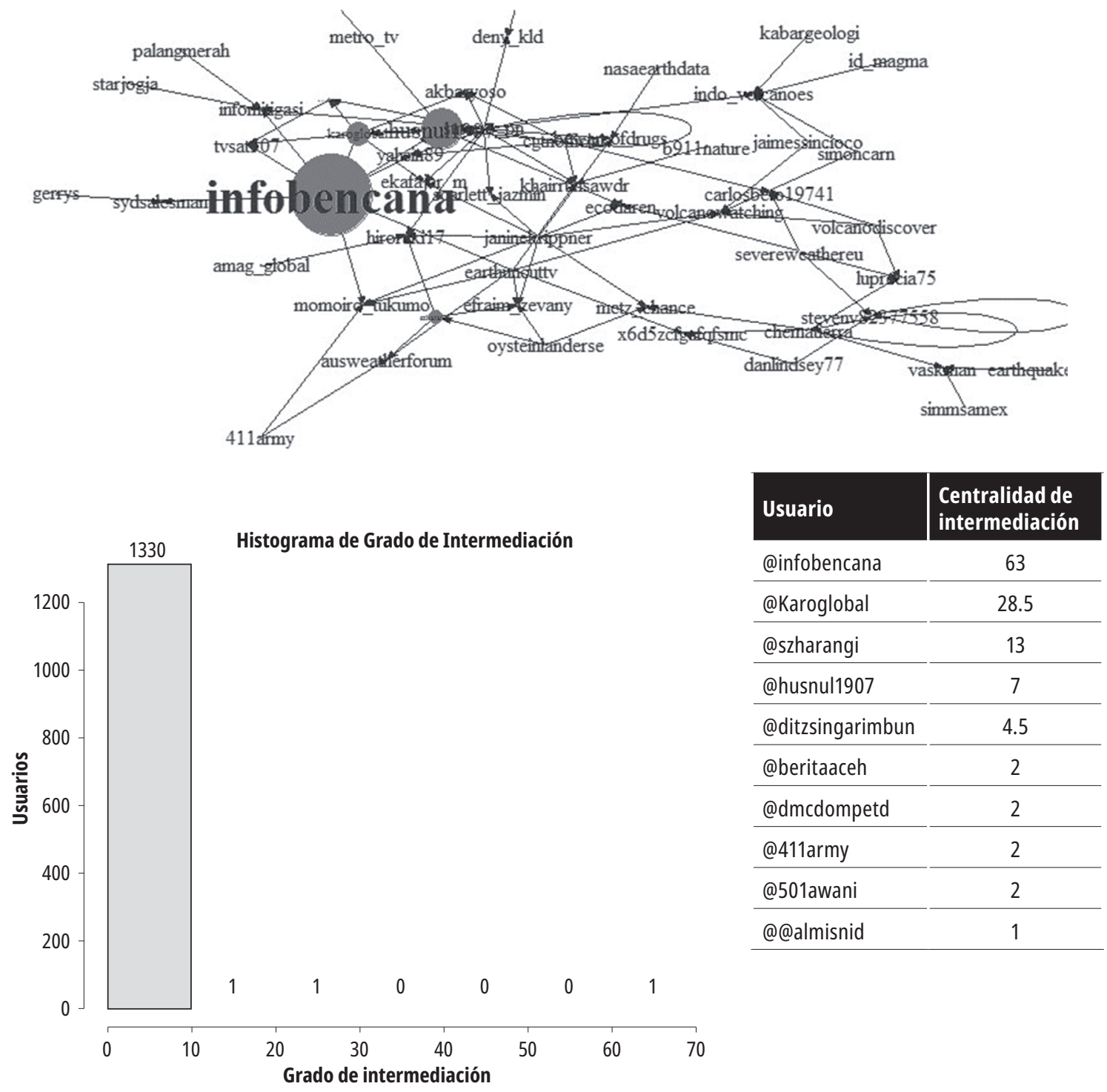

En la tabla 3 se presenta el análisis de los primeros 10 usuarios según la métrica de valor propio. El usuario @sutopo_pn tiene la mayor centralidad por la cantidad de sus publicaciones tanto propias como compartidas, y por eso ejerce un efecto importante en los usuarios más centrales de la red. Es de resaltar que hay una fuerte participación de usuarios del ámbito técnico y académico (Chematierra, Janinekrippner, f_a_h_a_d ), lo cual no fue identificado en [15]. 
Tabla 3. Top 10 centralidad de vector propio (Eigenvector) y distribución de grado
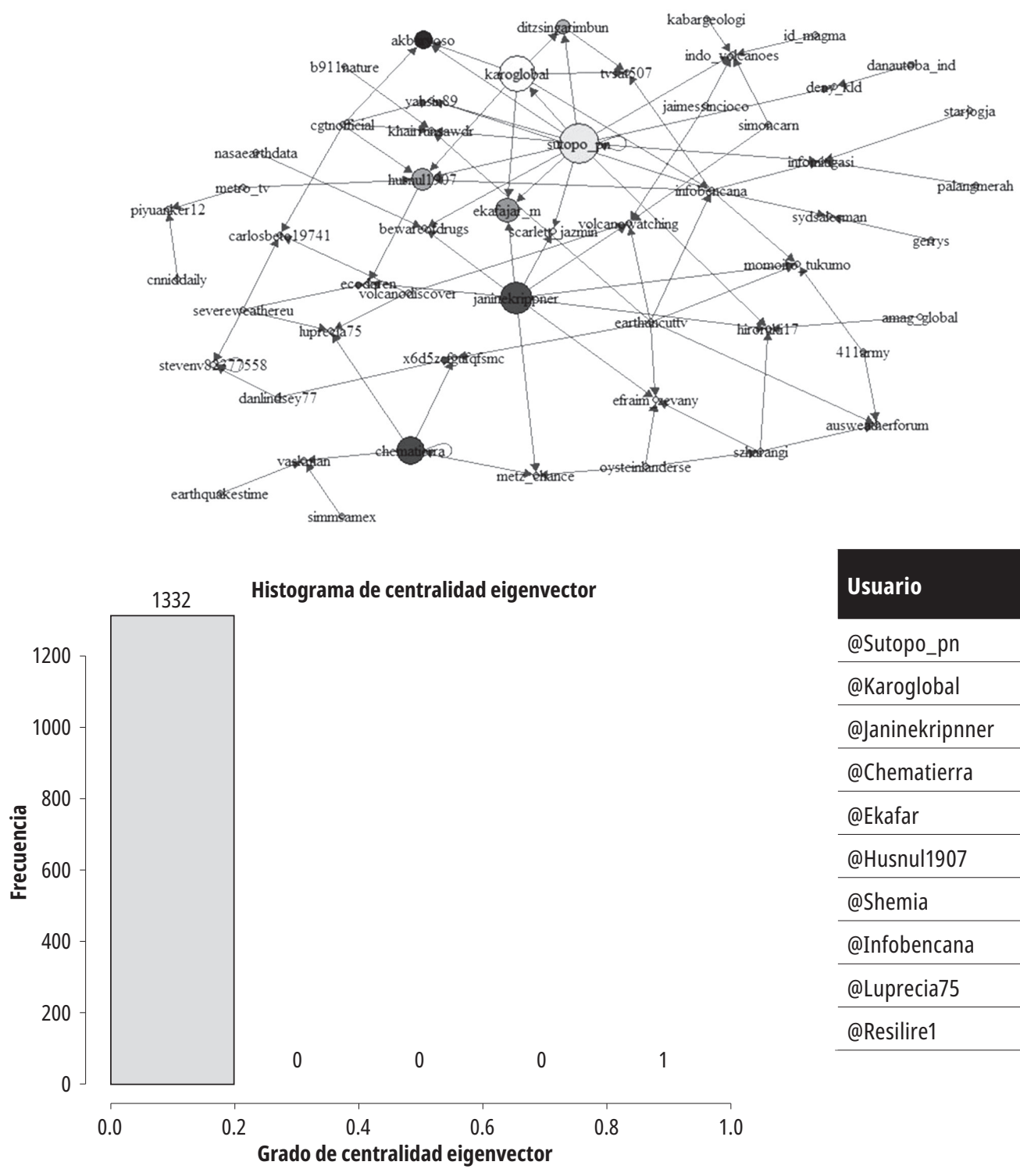

\begin{tabular}{l|c} 
Usuario & $\begin{array}{c}\text { Centralidad de } \\
\text { Eigenvector }\end{array}$ \\
\hline @Sutopo_pn & 1 \\
\hline @Karoglobal & 0.1590 \\
\hline @Janinekripnner & 0.1445 \\
\hline @Chematierra & 0.1396 \\
\hline @Ekafar & 0.1287 \\
\hline @Husnul1907 & 0.1280 \\
\hline @Shemia & 0.1279 \\
\hline @Infobencana & 0.1101 \\
\hline @Luprecia75 & 0.1004 \\
\hline @Resilire1 & 0.0766 \\
\hline
\end{tabular}

Como se dijo anteriormente, otros elementos que se analizan en este estudio son los hashtags. En la figura 5 se muestra el diagrama general de la red de hashtags, representados por los nodos. Las aristas representan la aparición simultanea de dos hashtags diferentes en el mismo trino. Este grafo incluye 1428 enlaces y 939 nodos. Como se muestra en la figura la red esta formada por grupos de hashtags fuertemente conectados entre sí.
En la tabla 4 se observan los principales hashtags de acuerdo con la centralidad de grado: \#sinabung, \#volcano $y$ \#Indonesia, entre otros. Están asociados a la localización del evento (\#Sinabung 236, \#Indonesia 81, \#Sumatra 48, \#Gunungsinabung 30, \#Indonesia's 23, \#Mountsinabung 21), la naturaleza (\#Volcano 92, \#Eruption 50, \#Erupsi 16) o los medios de comunicación (\#News 21). 


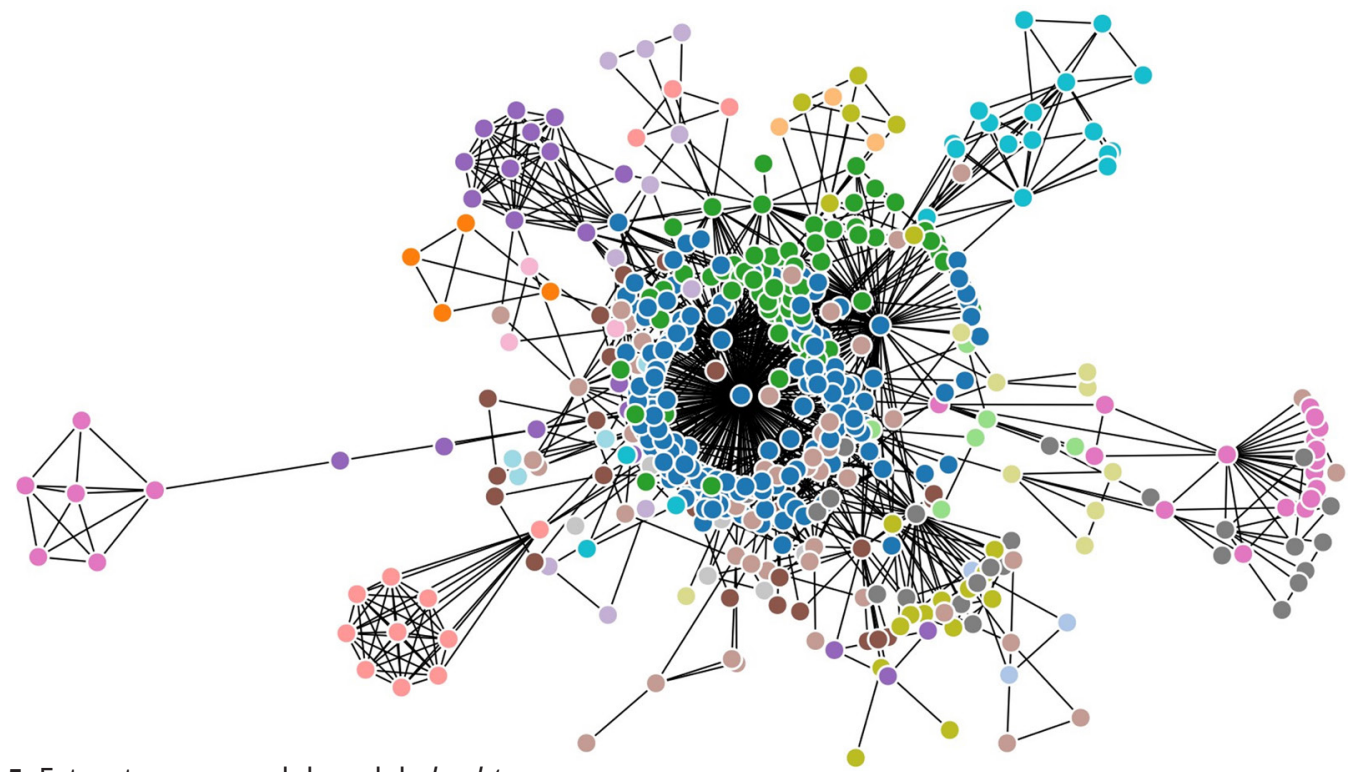

Figura 5. Estructura general de red de hashtags

Tabla 4. Top 10 centralidad de grado
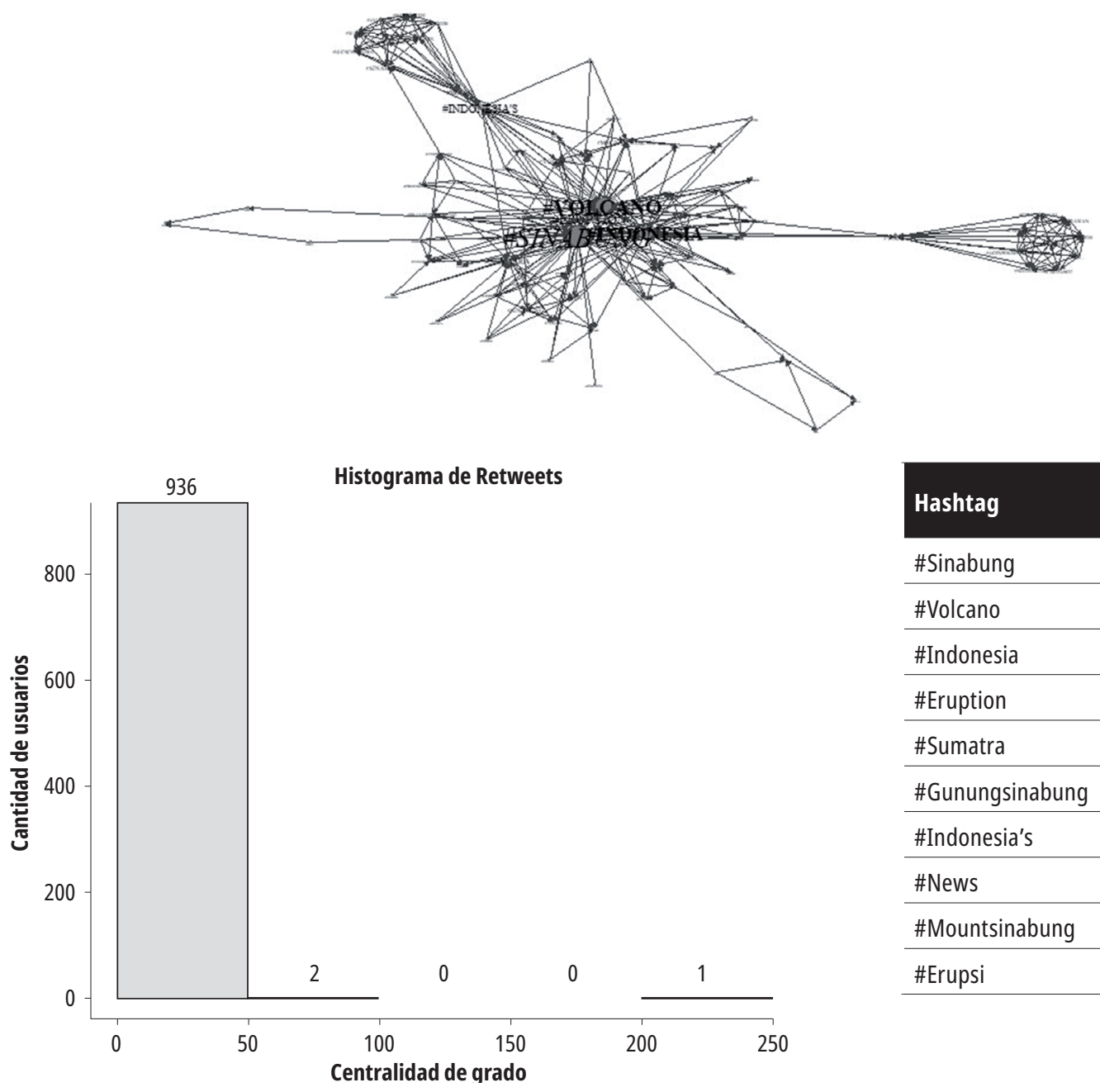

\begin{tabular}{l|c} 
Hashtag & $\begin{array}{c}\text { Centralidad } \\
\text { de grado }\end{array}$ \\
\#Sinabung & 236 \\
\hline \#Volcano & 92 \\
\hline \#Indonesia & 81 \\
\hline \#Eruption & 50 \\
\hline \#Sumatra & 48 \\
\hline \#Gunungsinabung & 30 \\
\hline \#Indonesia's & 23 \\
\hline \#News & 22 \\
\hline \#Mountsinabung & 21 \\
\hline \#Erupsi & 16 \\
\hline
\end{tabular}


En la tabla 5 se muestran los nodos con mayor centralidad de intermediación. Se observan los mismos hashtags obtenidos con la centralidad de grado, salvo \#Prayforsinabung. Esto se debe a que tal etiqueta no fue utilizada habitualmente en los mensajes consolidados, pero apareció acompañando hashtags frecuentes. A nivel general los resultados a partir de estas medidas muestran que la red fue usada para reportar la situación y expresar apoyo y solidaridad hacia la población afectada, tal y como se concluye en [15].
Con respecto a las URL se generó un grafo con dos tipos de nodos, uno que representa a los usuarios y otro a aquellas, lo que incluye un total de 920 vértices. Por otro lado, la acción de citar una URL por un usuario en un trino está representada por las aristas del grafo, lo que incluye un total de 1105 enlaces. En la figura 6 se muestra el diagrama general de la red de URL, de modo que se pueden ver las fuentes más compartidas por los usuarios. En dicha tabla se visualizan además los 10 nodos con mayor centralidad de grado. En ese sentido se

Tabla 5. Top 10 centralidad de intermediación

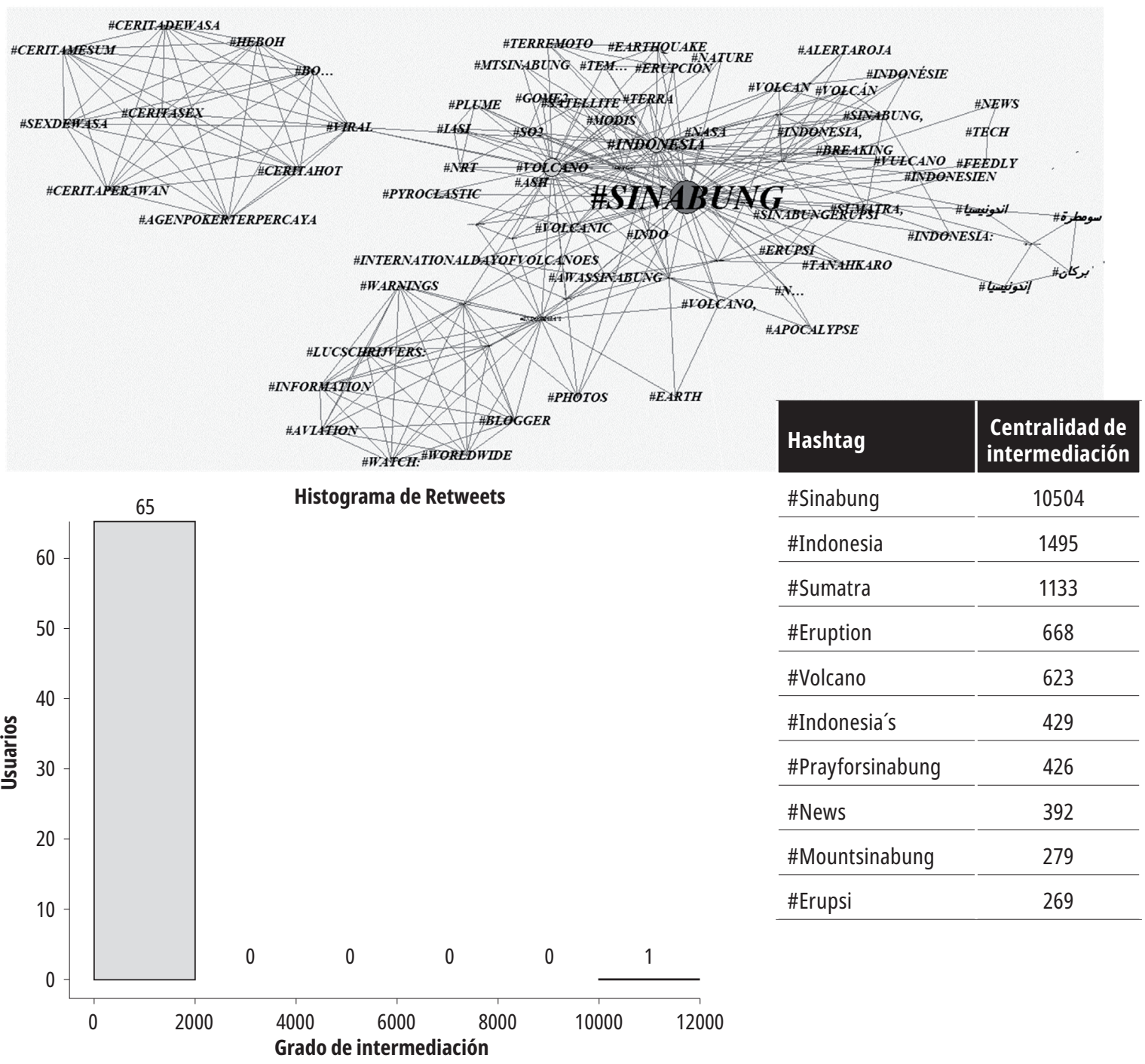


observan las páginas web más relevantes - como Twitter, Facebook e Instagram - participando como medios de comunicación. Youtube fue otro medio de comunicación representativo, al ser un sitio web popular dedicado a compartir vídeos del evento en tiempo real. Sin embargo, igualmente se identifican URL como viva.com, gizmodo.com, mashable.com y news.dekit.com, que son portales de noticias en línea. También se identificaron usuarios que compartieron publicaciones referenciadas con bit.ly, que redirigía información de otras páginas web. Así mismo, se registraron paginas como feeds.feedburner.com, un gestor que ofrece la posibilidad de crear una lista de suscriptores para que reciban por correo electrónico todo tipo de noticias.

Por otro lado, la distribución de grado de los nodos mostrada en la tabla 6 está sesgada a la derecha, lo que indica una mayor cantidad de nodos con baja centralidad de grado: 1102 nodos (URL y usuarios) tuvieron una centralidad en este sentido de entre 0 y 50 y solo 3 mostraron una entre 50 y 250. Es de resaltar que las URL no fueron tenidas en cuenta en el trabajo presentado en [15].

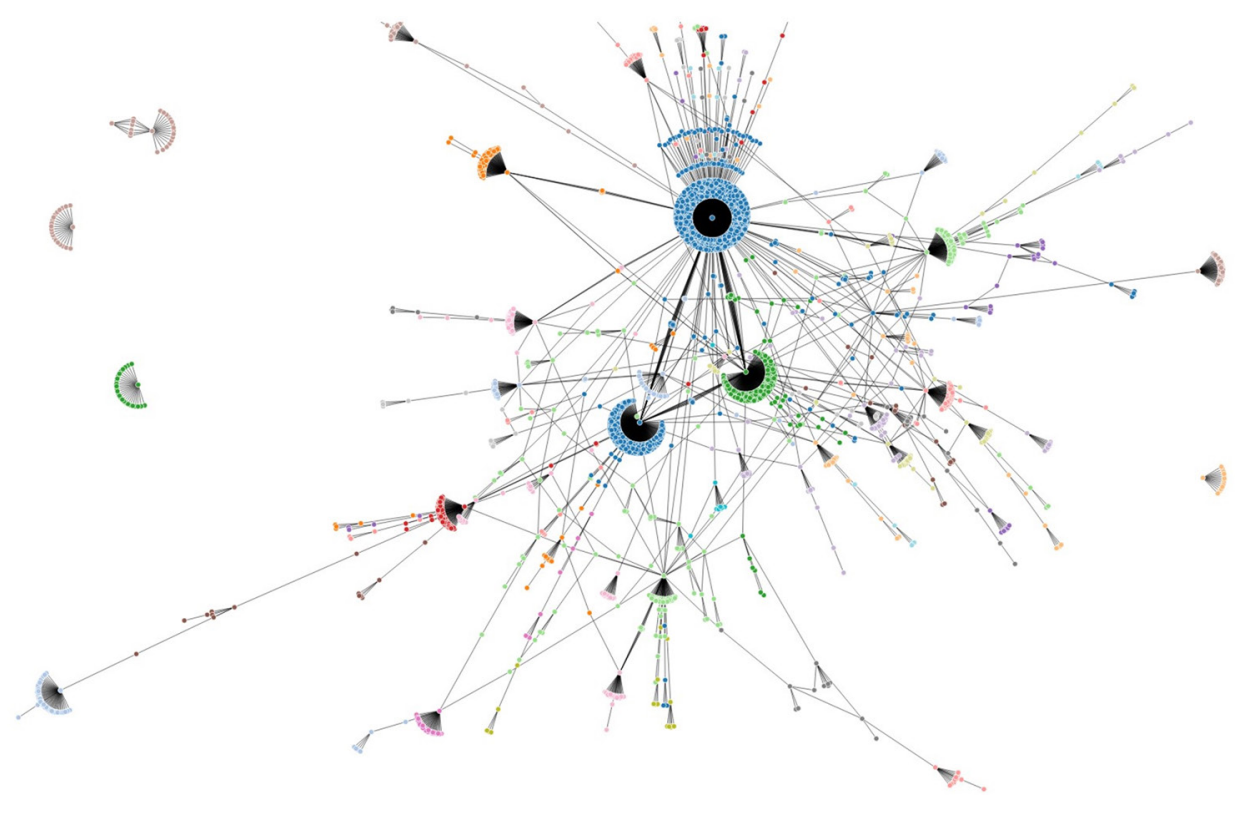

Figura 6. Estructura general de red de URL 
Tabla 6. Top 10 principales nodos con mayor centralidad de grado (degree)

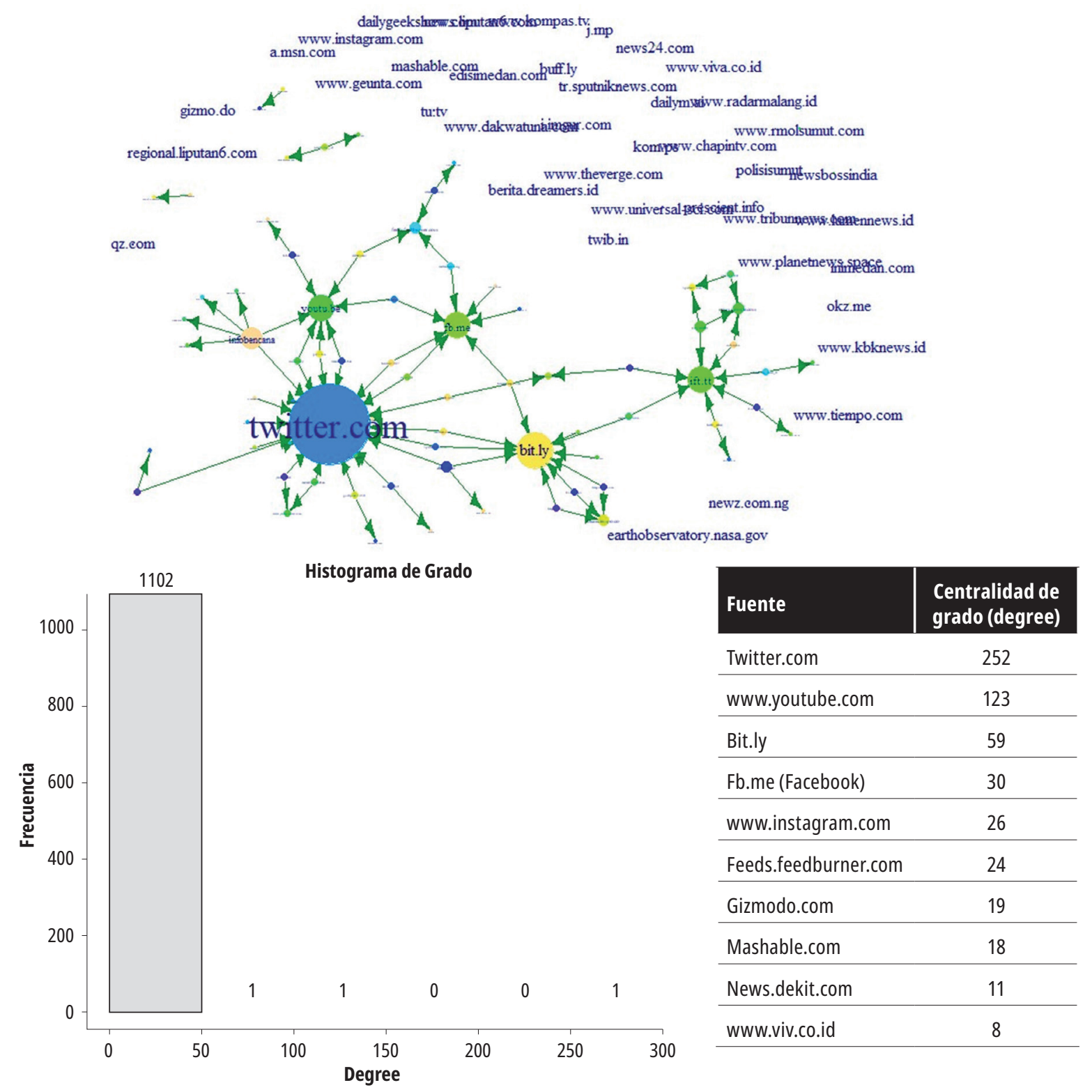

Como complemento al análisis topológico realizado se construyó la tabla 7 con algunas características básicas de las tres redes estudiadas. Los datos utilizados en las redes anteriores indican que el diámetro generado por cada red es relativamente pequeño respecto al número de nodos y sus enlaces. Este fenómeno se conoce comúnmente como red de mundo pequeño porque no todos los nodos son vecinos entre sí, pero la mayoría son accesibles a partir de cualquier otro nodo. 
Tabla 7. Comparación de las métricas de centralidad de las redes analizadas

\begin{tabular}{lcccc}
\hline & Retweets & Hashtags & URL \\
\hline Nodos & 282 & 939 & 920 \\
\hline Enlaces & 264 & 1428 & 1105 \\
\hline Diámetro & 13 & 8 & 10 \\
\hline Densidad & 0.003319753 & 0.001621287 & 0.000754147 \\
\hline Reciprocidad & 0 & 0 & 0 \\
\hline Transitividad & $0.075 \%$ & $10.68 \%$ & 0 \\
\hline $\begin{array}{l}\text { Longitud media } \\
\text { de Camino }\end{array}$ & 1 & 2 & 1 \\
\hline Grado medio & 2 & 3 & 2 \\
\hline
\end{tabular}

En cuanto a la densidad de los grafos, tal como se vio en el análisis de cada red, tiene valores muy bajos, lo que los convierte en grafos dispersos. El grafo de los retweets tiene el valor mas alto de densidad, seguido por el de hashtags y el de URL. Por otra parte, los valores para la reciprocidad son nulos en todos los grafos generados. Esto ocurre a causa de la configuración de la red ya que las relaciones de los grafos de retweets, hashtags y URL suelen estar orientadas en una sola dirección, es decir, un usuario puede hacer a otro retweet, mención y publicación. Por su parte, la red de hashtags se diferencia de los otros grafos en el promedio de grado, con un valor de 3 enlaces; una longitud de camino media de 2 y la transitividad de $10.68 \%$, los valores mas altos en cada métrica. De forma análoga, la red de hashtags es la de menor diámetro, con valor de 8 .

\section{Conclusiones}

En el presente estudio se aplica una metodología para la identificación y análisis de los actores en la red social Twitter utilizando un enfoque de ARS en un caso de desastre. Para la implementación de la metodología se eligió como caso de estudio la erupción del volcán Sinabung en 2018. Para el análisis de las partes involucradas en la comunicación sobre el desastre se generaron tres grafos (retweets, hashtags y URL). Las medidas estadísticas proporcionaron información sobre la estructura de cada red, e indicaron bajas medidas de densidad y conectividad. Esto es característico de redes dispersas, lo cual es coman en un medio como Twitter. A partir de las distribuciones de centralidad todas presentaron un sesgo a la derecha, lo que indica que la mayoría de nodos tenían un bajo grado de centralidad, pero un pequeño número de nodos mostró una centralidad alta. Se concluye, a partir de los datos consolidados, que Twitter no fue utilizado como un medio de comunicación de doble vía, es decir, de mensaje-respuesta. Esta afirmación se refuerza principalmente al considerar las medidas de reciprocidad nulas en los tres grafos. A nivel general la red se usó para reportar la situación y expresar apoyo y solidaridad hacia la población afectada, tal y como se concluye en un estudio de referencia [15].

Es de resaltar que hay una fuerte participación de usuarios del ámbito técnico y académico (Chematierra, Janinekrippner, f_a_h_a_d), lo cual no fue identificado en un estudio anterior [15]. Para futuras investigaciones se recomienda conducir estudios empíricos en la región, que validen los efectos observados en el trabajo aquí presentado aproximándose a la comunidad. Eso permitiría, por ejemplo, determinar una posible ruta de integración de la metodología utilizada en este estudio con actividades para la gestión de desastres.

\section{Referencias}

[1] J. Holguín-Veras, M. Jaller, L. N. Van Wassenhove, N. Pérez y T. Wachtendorf, "On the unique features of post-disaster humanitarian logistics", J. Oper. Manag., vol. 30, n. ${ }^{\text {s }} 7-8$, pp. 494-506, nov. 2012. DOI: https:// doi.org/10.1016/j.jom.2012.08.003

[2] H. Rodríguez, W. Díaz, J. M. Santos y B. E. Aguirre, "Communicating risk and uncertainty: Science, technology, and disasters at the crossroads", en Handbook of Disaster Research; H. Rodríguez, E. L. Quarantelli y R. R. Dynes, eds., Nueva York: Springer, 2007, pp. $476-$ 488. DOI: https://doi.org/10.1007/978-0-387-323534_29

[3] K. Carley, M. Malik, P. Landwehr, J. Pfeffer y M. Kowalchuck, "Crowd sourcing disaster management: The complex nature of Twitter usage in Padang Indonesia”, Saf. Sci., vol. 90, pp. 48-61, dic. 2016. DOI: https://doi. org/10.1016/j.ssci.2016.04.002

[4] M. R. Nair, G. R. Ramya y P. B. Sivakumar, "Usage and analysis of Twitter during 2015 Chennai flood towards disaster management", Procedia Compu. Sci., vol. 
115, pp. 350-358, 2017. DOI: https://doi.org/10.1016/j. procs.2017.09.089

[5] H. Gao, G. Barbier, R. Goolsby y D. Zeng, "Harnessing the crowdsourcing power of social media for disaster relief”. IEEE Intell. Syst., vol. 26 , n. ${ }^{\circ} 3$, pp. 10-14, my.jun. 2011. DOI: https://doi.org/10.21236/ADA581803

[6] P. M. Landwehr y K. M. Carley, "Social media in disaster relief”, en Data mining and knowledge discovery for Big Data; W. W. Chu, ed., Berlín/Heidelberg: Springer, 2014, pp. 225-257. DOI: https://doi.org/10.1007/978-3642-40837-3_7

[7] M. E. Keim y E. Noji, "Emergent use of social media: A new age of opportunity for disaster resilience", Am. J. Disaster Med., vol. 6, n. ${ }^{\circ}$ 1, pp. 47-54, 2011. DOI: https://doi.org/10.5055/ajdm.2011.0044

[8] K. Zahra, M. Imran y F. O. Ostermann, “Automatic identification of eyewitness messages on Twitter during disasters", Inform. Process. Manag.,

vol. 57, n. ${ }^{\circ}$, pp. 102-107, en. 2020. DOI: https://doi.org/10.1016/j.ipm.2019.102107

[9] B. Takahashi, E. C. Tandoc y C. Carmichael, "Communicating on Twitter during a disaster: An analysis of tweets during typhoon Haiyan in the Philippines", Comput. Human Behav., vol. 50, pp. 392-398, sep. 2015. DOI: https://doi.org/10.1016/j.chb.2015.04.020

[10] B. F. Liu, J. D. Fraustino y Y. Jin, "How disaster information form, source, type, and prior disaster exposure affect public outcomes: Jumping on the social media bandwagon?", J. Appl. Commun. Res., vol. 43, n. ${ }^{\circ}$ 1, pp. 44-65, en. 2015. DOI: https://doi.org/10.1080/0090988 2.2014 .982685
[11] J. Scott y P. J. Carrington, The Sage handbook of social network analysis. Londres: Sage, 2011.

[12] M. A. Smith, L. Rainie, B. Shneiderman e I. Himelboim, "Mapping Twitter topic networks: From polarized crowds to community clusters", Pew Res. Cent., vol. 20, pp. 1-56, 2014.

[13] N. Pourebrahim, S. Sultana, J. Edwards, A. Gochanour y S. Mohanty, "Understanding communication dynamics on Twitter during natural disasters: A case study of Hurricane Sandy", Int. J. Disast. Risk Red., vol. 37, pp. 101-176, jul. 2019. DOI: https://doi.org/10.1016/j. ijdrr.2019.101176

[14] M. Oliveira y J. Gama, "An overview of social network analysis", Wiley Interdiscip. Rev. Data Min. Knowl. Discov., vol. 2, n. ${ }^{\circ}$ 2, pp. 99-115, mar. 2012. DOI: https://doi.org/10.1002/widm.1048

[15] A. T. Chatfield, C. G. Reddick, D. I. Inan y U. Brajawidagda, "E-government, social media, and risk perception communication at the edge of disaster", en Proceedings of the 15th Annual International Conference on Digital Government Research, 2014, pp. 153-162. DOI: https://doi.org/10.1145/2612733.2612752

[16] S. Wasserman y K. Faust, Social network analysis: methods and applications. Cambridge: CUP, 1994. DOI: https://doi.org/10.1017/CBO9780511815478

[17] D. Knoke y S. Yang, Social network analysis. Thousand Oaks, CA: Sage, 2008. DOI: https://doi. org/10.4135/9781412985864

[18] Volcano Discovery, s. f. Diponible en https://tinyurl. $\mathrm{com} / \mathrm{rbl} 2 \mathrm{a}$ 7 
